\title{
On X-ray emission lines from active galactic nuclei and disk models
}

\author{
A. Čadežํ, M. Brajnik ${ }^{1}$, A. Gomboc ${ }^{1}$, M. Calvani ${ }^{2}$, and C. Fanton ${ }^{2}$ \\ 1 Department of Physics, University of Ljubljana, Jadranska 19, 6100 Ljubljana, Slovenia \\ 2 INAF - Astronomical Observatory of Padova, vicolo Osservatorio 5, 35122 Padova, Italy
}

Received 21 October 2002 / Accepted 7 January 2003

Abstract. An efficient numerical code to calculate line profiles from warped disks around nonrotating black holes is presented. Extensive numerical experiments suggest a method making it possible to distinguish between line profiles belonging to flat and warped accretion disks. The extension of our code to rotating black holes is briefly discussed.

Key words. accretion, accretion disks - black hole physics - galaxies: active

\section{Introduction}

Broad X-ray $\mathrm{Fe} \mathrm{K}_{\alpha}$ emission lines, first discovered by ASCA, are considered as the best clue to the presence of massive black holes in active galactic nuclei (AGN), see e.g. Fabian et al. (1995, 2000, 2001), Reynolds \& Nowak (2002). Line profile data provided by ASCA (Nandra et al. 1997), Chandra and XMM (e.g. Reeves et al. 2001) satellites are very often analyzed in the framework of flat, X-ray corona illuminated, optically thick accretion disks around nonrotating or rotating black holes. In the case of ASCA data, most lines could be fit with such a model, assuming also a simple radial power law emissivity in the proper frame of the disk. With the new higher signal to noise data coming from XMM, it is becoming apparent that such a model may soon prove to be too simple to fit with the data. Different modifications of the simple thin flat disk power law emissivity model have been proposed in the literature as candidates for better description of the environment around the central black hole of an AGN (warped disks, toroids, illumination by points sources on and off axis, shadowing by tori, widening of lines by Compton scattering etc., see e.g. Kojima \& Fukue 1992; Usui et al. 1998; Bachev 1999; Blackman 1999; Yu \& Lu 2000; Hartnoll \& Blackman 2000, 2001; Karas et al. 2001; Martocchia et al. 2000, 2002). All such proposals are based on sound arguments. However, it is also clear that introducing new parameters always improves the agreement of the theoretical proposal with data. Thus, it might be useful to gauge different theoretical models with respect to the simple flat disk model, allowing the emissivity in the proper disk frame to be any positive function of the radius $r$ best describing the measured line profile. In this way we are able to present some characteristics of a given input model (as for example of warped

Send offprint requests to: M. Calvani, e-mail: calvani@pd.astro.it disks) in a systematic way. We find for example, that a moderately warped disk can be fit by such a flat disk model, but the fit requires an increasingly complex emissivity profile as the warp increases. A flat disk model can also produce different ratios of the blue to red wing of the line profile, but a warp in a disk can reproduce extreme ratios with a much more natural emissivity law. Moreover, the correlation between the blue and red peak variability can be modelled by a complicated change in the radial emissivity law, but it finds a much more natural explanation in terms of a precessing warp.

The present analysis focuses mostly on X-ray emission lines coming from the innermost regions of accretion disks, however, the tools we developed can be applied as well to investigate emission lines originating further away from the black hole (optical emission lines, e.g. Calvani et al. 1997; Sulentic et al. 2000).

The plan of the paper is as follows: in Sect. 2 the numerical code to calculate line profiles from warped disks around non-rotating black holes is described. In Sect. 3 we present a collection of theoretical line profiles from warped disks with different parameters and compare them to the closest flat disk profiles. Section 4 is devoted to numerical experiments with warped disk line profiles in order to find a tool to distinguish between line profiles belonging to flat and warped disks. In Sect. 5 we discuss how to extend our results to rotating black holes and we present some preliminary results. Conclusions follow in Sect. 6.

\section{Generating theoretical line profiles for flat and warped disks around black holes}

The ray tracing technique for constructing images of accretion disks around black holes and producing their integrated line profiles has been described before in a number of articles (e.g. Fanton et al. 1997; Čadež et al. 1998 and references therein), 
so they need not be discussed in great detail. We do stress, however, the importance of numerical efficiency, since the multidimensional space of warped and obscured disks requires a large number of sample profiles to be adequately covered.

Our theoretical line profile numerical codes are based on warped disk models, describing thin, warped, optically thick disks around non-rotating black holes (for a justification of warped disks see e.g. Pringle 1996, 1997; Demianski \& Ivanov 1997; Quillen 2001; Lubow et al. 2002). The disk surface is supported by circular time-like geodesic orbits with varying inclinations to a chosen fixed axis centered to the black hole. For the shape of the warp we usually use the form proposed by Hartnoll \& Blackman (2000) who parametrize the twist free disk surface extending from $R_{\text {in }}=6 M$ (innermost stable circular orbit) to the to the freely specifiable outer radius $R_{\text {out }}$ by two parameters $a_{1}$ and $b$ in the following way:

$z(R, \phi)=a_{1} R_{\text {out }} 2^{(b-1) / 2}\left(\frac{R}{R_{\text {out }}}\right)^{b} \cos (\phi)$

Here $R, \phi, z$ are cylindrical coordinates related to the Schwarzschild coordinates $r, \theta, \phi$ in the usual way $(R=$ $r \sin \theta, z=r \cos \theta)$. Obviously $a_{1} 2^{(b-1) / 2}$ determines the angle between the normal to the inner and to the outer geodesic circle (if $a_{1}=0$, then the disk is flat), while $b$ can be thought of as determining the radius at which the transition occurs: the higher $b$ the further out and the steeper is the transition.

The twisted warp of Hartnoll \& Blackman (2000) has a fixed form, with only the amplitude $a_{2}$ of the warp as a parameter:

$z(R, \phi)=a_{2} \sqrt{\frac{R}{R_{\text {out }}}} \sin \left(\sqrt{\frac{10 R}{R_{\text {out }}}}\right) \cos \left(\phi-\sqrt{\frac{10 R}{R_{\text {out }}}}\right)$.

Before constructing the image of a warped disk around a black hole of mass $M^{1}$ one needs to specify also the source of illumination, the inclination $(\iota)$ of the disk plane to the observer and the longitude of the observer $\phi_{\text {obs }}=\Omega$ with respect to the coordinate system of the disk.

For the illumination source, following Hartnoll \& Blackman (2000), we use two point sources on the axis of the disk at the height $z= \pm h$, where $h$ is a free parameter (in units of black hole mass; often we use $h=10 M$ ), but we also have the choice to use the simple proper emissivity power law $\left(\epsilon(r) \propto r^{-q}\right)$.

To produce an image of the disk we proceed as follows:

- Define a rectangular mesh $P_{i j}=\left\{r_{i}, \theta(i, j), \phi_{j}\right\}$ of points in the disk surface, where $\phi_{j}=2 \pi j / N_{\phi}^{i}\left(i=0,1, \ldots N_{\phi}^{i}-1\right)$ and $r_{i}$ are Schwarzschild radial coordinates monotonously increasing with $i$. The explicit functional dependence of $r_{i}$ on $i$ is chosen in a logarithmic-like scale to compensate for the fast change of the redshift factor $g$ at small $r$.

- Construct the image of the rectangular mesh at the image plane $\left(Q_{i j}=\mathcal{M}\left[P_{i j}\right], Q_{i j}=\left\{x_{i j}, y_{i j}\right\}\right)$.

- If the length of the " $\phi$ " side connecting adjacent points $\left(Q_{i j}, Q_{i j+1}\right)$ in the image plane is larger then the length requirement (which is chosen in such a way as to produce a

\footnotetext{
${ }^{1}$ Geometric units $G=c=1$ are used throughout this text.
}

smooth image), insert a new point to the mesh $P_{i j}$, renumber the points, increase $N_{\phi}^{i}$ and repeat the procedure until all the " $\phi$ " sides meet the length requirement.

- Calculate the emissivity of the disk at the final mesh points.

- Triangulate ribbons $\left\{Q_{i j}, Q_{i+1 j}\right\}$ and mark the " $r$ " sides that cross the Einstein ring corresponding to the disk surface in opposition with respect to the observer.

- Calculate the values of $g$ (redshift factor), $I$ (radiance) and $\zeta$ (the projection of the Schwarzschild radial coordinate on the viewing direction) inside triangles by linear interpolation and "paint" the triangles in the image plane if the corresponding pixels have not been painted previously or the new $\zeta$ is closer to the observer than the one of the previous paint and if the triangles are not marked as crossing the Einstein ring.

- The corresponding line profile is obtained by simply adding all intensities in the image plane to the appropriate energy channels.

The efficiency of the above code is based on the fact that it is possible to find an almost analytic expression for the map $\mathcal{M}$, i.e. it is possible to analytically find constants of motion of a (lightlike) geodesic connecting any initial $\left(\mathcal{P}_{i}=\left\{r_{i}, \theta_{i}, \phi_{i}\right\}\right)$ and final $\left(\mathcal{P}_{f}=\left\{r_{f}, \theta_{f}, \phi_{f}\right\}\right)$ point in the Schwarzschild spacetime. The method, first discussed by Brajnik (1999) and elaborated by Gomboc (2001), can be described as follows. The initial and the final point determine the orbital plane of the geodesic from which the inclination of the orbital plane $(\varepsilon)$, the longitude of the ascending node $\left(\Omega_{\gamma}\right)$ and the angle $\alpha(<\pi)$ between $\mathcal{P}_{i}$ and $\mathcal{P}_{f}$ are easily found with the use of spherical trigonometry. The two remaining constants of the motion, the specific angular momentum of the photon $l_{\gamma} / E_{\gamma}=2 M / a$ and the longitude of periastron with respect to the orbital plane can be determined by a straightforward procedure, which is based on the following observations: in the orbital plane all lightlike geodesics reaching infinity ${ }^{2}$ can be arranged in two classes with respect to their angular momentum parameter $a\left(a=2 M E_{\gamma} / l_{\gamma}\right.$, where $E_{\gamma}$ and $l_{\gamma}$ are the energy and angular momentum of the photon respectively). Geodesics with $a<\frac{2}{3 \sqrt{3}}$ belong to the class we call $A$ and are scattered off the black hole. In the class $A$ it is convenient to introduce a parameter $\psi$ instead of $a$ :

$a=\frac{2}{3 \sqrt{3}} \sin \frac{\psi}{2}$

so that in terms of a true anomaly $\lambda$ reckoned from the initial point $\left(\mathcal{P}_{i}\right)$, the orbit equation can be expressed in the form:

$r(\lambda)=\frac{2 M}{u_{2}-\left(u_{2}-u_{3}\right) c n^{2}\left(\frac{\lambda}{2 n}-F\left(\chi_{i} \mid m\right) \mid m\right)}$,

where $u_{1}, u_{2}, u_{3}, m, n$ and $\chi_{i}$ are functions of the parameter $\psi$ only. They are:

$u_{1}=\frac{1}{3}\left(1+2 \cos \frac{\psi}{3}\right)$

$u_{2}=\frac{1}{3}\left(1+2 \cos \frac{\psi-2 \pi}{3}\right)$

${ }^{2}$ Lightlike geodesics with two ends inside the black hole are of no consequence for the present discussion. 
$u_{3}=\frac{1}{3}\left(1+2 \cos \frac{\psi+2 \pi}{3}\right)$

$m=\frac{u_{2}-u_{3}}{u_{1}-u_{3}}$

$n=\frac{1}{\sqrt{u_{1}-u_{3}}}$

$\cos \chi_{i}=\sqrt{\frac{u_{2}-u_{i}}{u_{2}-u_{3}}}$

Here $u_{i}=2 M / r_{i}, c n(x \mid m)$ is the Jacobi cosine amplitudinis and $F(x \mid m)$ the incomplete elliptic integral of the first kind. Elliptic integrals and functions are expressed in the notation adopted by Wolfram (1996).

Lightlike geodesics belonging to the second class, which we call $B$, reach from infinity and end beyond the horizon of the black hole. Their angular momentum parameter $a$ is greater than $\frac{2}{3 \sqrt{3}}$. In this case it is convenient to express $a$ in terms of a parameter $\mu$ :

$a=\frac{2}{3 \sqrt{3}} \cosh \mu$,

so that the orbit equation is expressed in the form:

$r(\lambda)=2 M\left[u_{1}+\frac{1}{n^{2}} \frac{1-c n\left(F\left(\chi_{i} \mid m\right)+\lambda / n \mid m\right)}{1+c n\left(F\left(\chi_{i} \mid m\right)+\lambda / n \mid m\right)}\right]^{-1}$.

Here again $u_{1}, m, n$ and $\chi_{i}$ are functions of $\mu$ only:

$u_{1}=\frac{1}{3}\left(1-2 \cosh \frac{2 \mu}{3}\right)$

$m=\frac{1}{2}\left(1-\frac{3 u_{1}-1}{2 \sqrt{u_{1}\left(3 u_{1}-2\right)}}\right)$

$n=\left[u_{1}\left(3 u_{1}-2\right)\right]^{-\frac{1}{4}}$

$\cos \chi_{i}=\frac{1-n^{2}\left(u_{i}-u_{1}\right)}{1+n^{2}\left(u_{i}-u_{1}\right)}$.

Finally in the special case when $a=\frac{2}{3 \sqrt{3}}$ the orbit equation becomes simply:

$r(\lambda)=\frac{2 M}{-\frac{1}{3}+\operatorname{Tanh}^{2}\left(\lambda / 2+\operatorname{ArcTanh} \sqrt{1 / 3+u_{i}}\right)}$.

The determination of $a$ now proceeds as follows. One first determines the class that the geodesic connecting $P_{i}$ and $P_{f}$ belongs to. To this end consider the point $P_{f}$ in the polar plane $(r, \lambda)$ in Fig. 1.

The two critical geodesics (the prograde and the retrograde one) passing through $P_{f}$ with $a=\frac{2}{3 \sqrt{3}}$ define three regions denoted by $I, I I$ and $I I I$. A point $P_{i}$ laying in the interior of the circle $r=3 M-$ region $I I I-$ can only be reached by a geodesic of type $B$. If $P_{i}$ is in the region $I I$, then the most direct geodesic to reach it is type $B$, which always remains in region $I I$. However, a point $P_{i}$ in region $I I$ can also be reached from $P_{f}$ by geodesics of type $A$ with the angular momentum very close to the critical value, so that it winds about the circle $r=3 M$ on the outside before crossing the boundary $I-I I$. For such a geodesic the true anomaly $\lambda$ describes an angle $(2 N \pi-\alpha)$, where $\alpha<\pi$ and $N$ is the winding number $\geq 1$ for boundary crossing geodesics of type $A$. If $P_{i}$ is in the region $I$, then it can only be reached by a geodesic of type $A$ with $N \geq 0{ }^{3}$.

After determining the type of the geodesic and the angle $\Delta \lambda$ that the true anomaly describes from $P_{f}$ to $P_{i}$, one chooses the appropriate geodesic equation and expresses the cosine amplitudinis at both points.

Assume that one wants to find a geodesic of type $A$ that goes from $r_{\mathrm{f}} \rightarrow \infty$ to $r_{i}$. At $P_{i}$ one obtains:

$c n\left(F\left(\chi_{i} \mid m\right) \mid m\right)=\sqrt{\frac{u_{2}-u_{i}}{u_{2}-u_{3}}}$,

similarly at $P_{f}$ :

$c n\left(F\left(\chi_{i} \mid m\right)-\frac{\Delta \lambda}{2 n} \mid m\right)=\sqrt{\frac{u_{2}}{u_{2}-u_{3}}}$.

These are two equations for two unknowns $\psi$ (which is the argument of $m, n, u$ 's) and of $\chi_{i}$. Using the Jacobi elliptic functions addition theorem, $\chi_{i}$ can be eliminated as follows: introduce

$v=F\left(\chi_{i} \mid m\right)-\frac{\Delta \lambda}{2 n}$

$z=F\left(\chi_{i} \mid m\right)$

and write:

$$
\begin{aligned}
c n(v-z \mid m) & =c n\left(\frac{\Delta \lambda}{2 n}\right) \\
& =\frac{c n(v \mid m) c n(z \mid m)+s n(v \mid m) \operatorname{sn}(z \mid m) d n(v \mid m) d n(z \mid m)}{1-m s n^{2}(v \mid m) s n^{2}(z \mid m)} .
\end{aligned}
$$

All the elliptic functions on the right hand side of the above equation can be expressed via Eqs. (18) and (19) in terms of $u_{1}, u_{2}, u_{3}$, which are known functions of $\psi$, listed below for convenience:

$c n^{2}(v \mid m)=\frac{u_{2}}{u_{2}-u_{3}}$

$s n^{2}(v \mid m)=\frac{-u_{3}}{u_{2}-u_{3}}$

$d n^{2}(v \mid m)=\frac{u_{1}}{u_{1}-u_{3}}$

$c n^{2}(z \mid m)=\frac{u_{2}-u_{i}}{u_{2}-u_{3}}$

$\operatorname{sn}^{2}(z \mid m)=\frac{u_{i}-u_{3}}{u_{2}-u_{3}}$

$d n^{2}(v \mid m)=\frac{u_{1}-u_{i}}{u_{1}-u_{3}}$

Thus Eq. (22) is a nonlinear equation for $\psi$, which can be effectively solved by the secant method. $\chi_{i}$ is then easily obtained from Eq. (18) and the components of tangent vectors to the geodesic at $P_{f}$ follow in a straightforward way. For geodesics of type $B$ the method is identical.

\footnotetext{
${ }^{3}$ The normal is always oriented so that $\lambda$ increases from $P_{f}$ to $P_{i}$.
} 


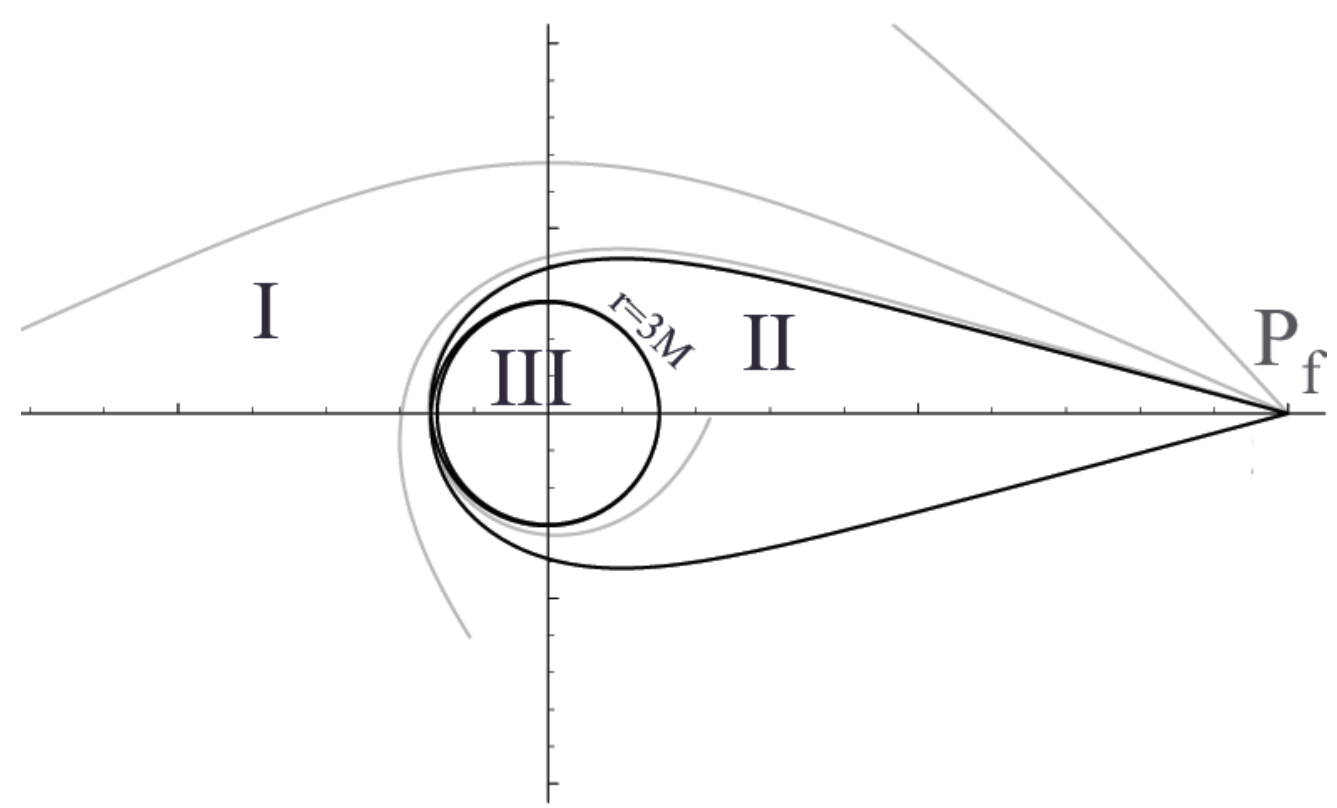

Fig. 1. The critical geodesics (heavy lines) through $P_{f}$ divide the orbital plane in three regions. Some geodesics of type $A$ through $P_{f}$ are also shown (gray lines).

\section{The effect of warps on line profiles}

It is obvious, and we elaborate on this issue in the next section, that a given stationary line profile does not at all specify uniquely the emissivity in the disk unless one makes further assumptions, as for example that the disk is flat and that the emissivity depends only on the radial coordinate. Thus, a given line profile can lead to different heuristic disk models and only the plausibility of the corresponding emissivity will be finally appreciated through a physical disk model. Therefore, we suggest it is useful to gain understanding of what are the main distinguishing features between different disk models by presenting and discussing some theoretical disk images and their line profiles.

Figure 2 shows a series of images of a mildly warped disk ( $a_{1}=0.1, b=3$ ) illuminated by two point sources on the axis at $h= \pm 10 M$ above the disk plane. The outer radius of the disk is $R_{\text {out }}=40 \mathrm{M}$ and the observer is at $\iota=30^{\circ}$ above the disk plane; images are arranged in order of increasing $\Omega$. Corresponding $\mathrm{Fe} \mathrm{K}_{\alpha}$ line profiles are also shown to the right of each image. It is apparent that in this case the extremities of the line do not change appreciably with $\Omega$, since the innermost region of the disk, which determines the spread of the line, is never hidden to the observer or shadowed by the warp. However one notes that the ratio of the blue to red peak varies considerably even for this moderate warp. By comparing the pictures and the lines, this is recognized mostly as the consequence of shadowing either the blue or red part of the visible image. A square array of line profiles of another moderately warped disk $\left(a_{1}=0.1, b=3\right.$, $\left.R_{\text {out }}=100 M\right)$ is presented in Fig. 3 together with flat disk line profiles (in light gray) observed from the same inclination (we assume the rest energy of the line to be $6.4 \mathrm{keV}$ ). We would like to call attention to the fact that for a moderate warp the blue edge of the line is not affected by warping. This is understood, by the same argument made before, that the innermost disk regions are visible to the observer. However, this is not true at high inclinations: $\iota=80^{\circ}, \Omega=240^{\circ}, 300^{\circ}$ where the warp hides predominantly the central blue parts of the disk.

A similar array of twisted disk $\left(a_{2}=0.3, R_{\text {out }}=100 M\right)$ line profiles is shown in Fig. 4 together with flat disk profiles. The blue edge no longer seems to follow the corresponding Schwarzschild profile. This is easily understood as now the inner part of the disk is tilted to the disk plane so that its inclination to the observer depends on the point of view $(\Omega)$ and is different than that of the average disk plane. Shadowing again becomes important at high inclinations and its effects are most pronounced for $\iota=80^{\circ}, \Omega=0^{\circ}, 300^{\circ}$.

\section{Line profiles and disk emissivity}

In analyzing the low signal to noise ASCA data it was standard to fit $\mathrm{Fe}_{\alpha}$ emission lines in the framework of flat accretion disks with a power law emissivity. However, even with these data it is often found that one or a number of data channels hold a value that is considerably different from any model prediction. Therefore, following Lucy (1974), Mannucci et al. (1992) and Dabrowski et al. (1997), we proposed a new method for inverting the integral equation

$F(E)=\int \varepsilon(r) K\left(E, R \mid \iota_{m}, a_{\mathrm{rot}}\right) \mathrm{d} R$

which connects the disk emissivity $\varepsilon(r)$ to the line profile in the framework of the flat disk model. In this way we wanted to test if it is possible to find a radial emissivity profile that would provide more plausible line fits (Čadež et al. 2000). We demonstrated that ASCA line profiles can generally be fitted to such a model. The inclination of the disk $\left(\iota_{m}\right)$ was found to be reasonably well specified by the fit, however the angular momentum of the black hole $\left(a_{\text {rot }}\right)$ remains undetermined, except in the case of MCG-6-30-15, where the large extent of the line 

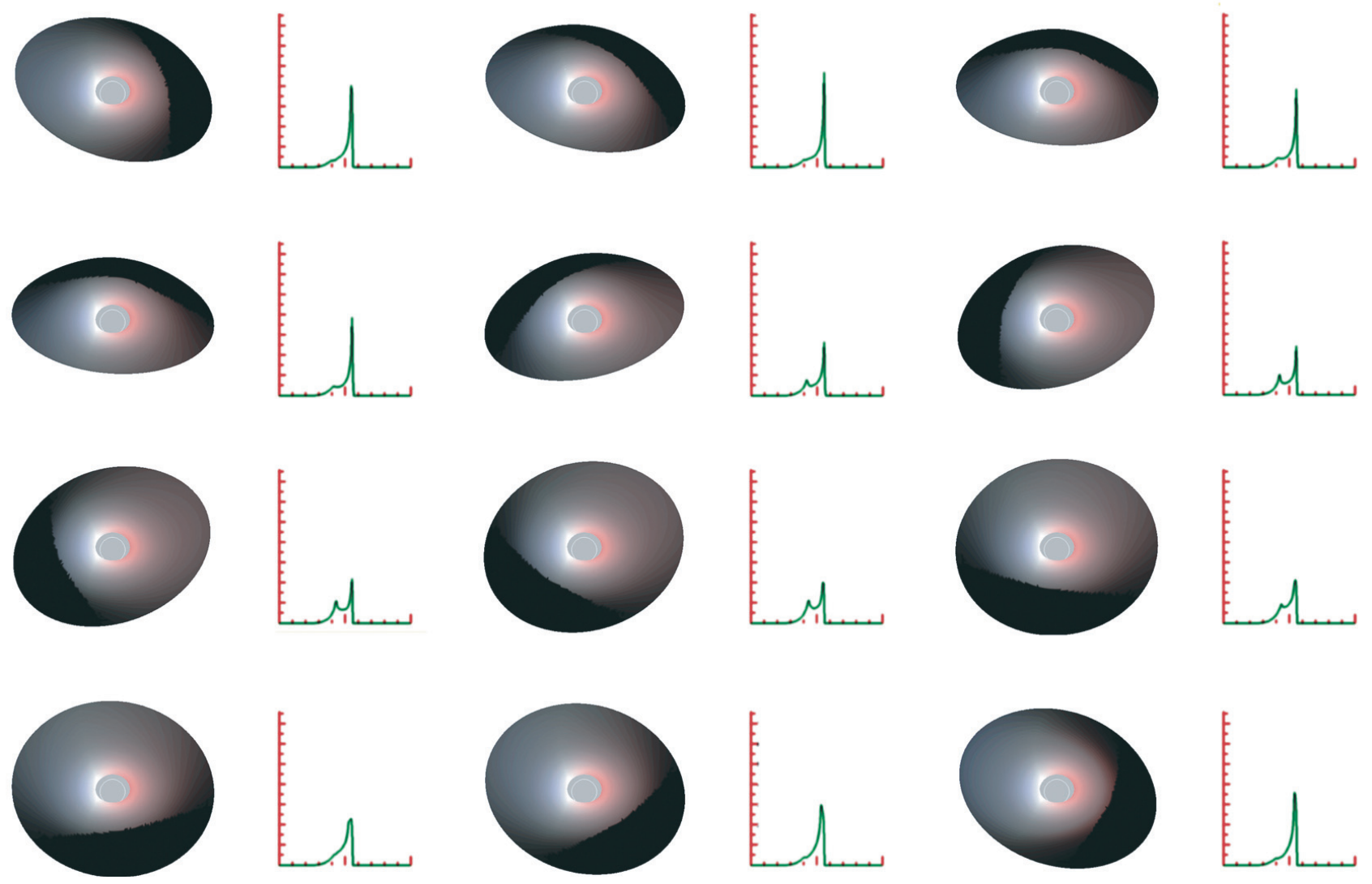

Fig. 2. Images of a mildly warped disk $\left(a_{1}=0.1, b=3, R_{\text {out }}=40 M\right)$, illuminated by two point sources at $h= \pm 10 M$, seen from the inclination $\iota=30^{\circ}$ for $\Omega=0^{\circ}$ (top left) to $\Omega=330^{\circ}$ (bottom right) in steps of $30^{\circ}$. The hue of the color corresponds to the redshift (blueshift) and the intensity of the colors is in a semi-log scale. The corresponding line profiles are also shown as intensity vs. redshift $g$ with $0 \leq g \leq 2$.
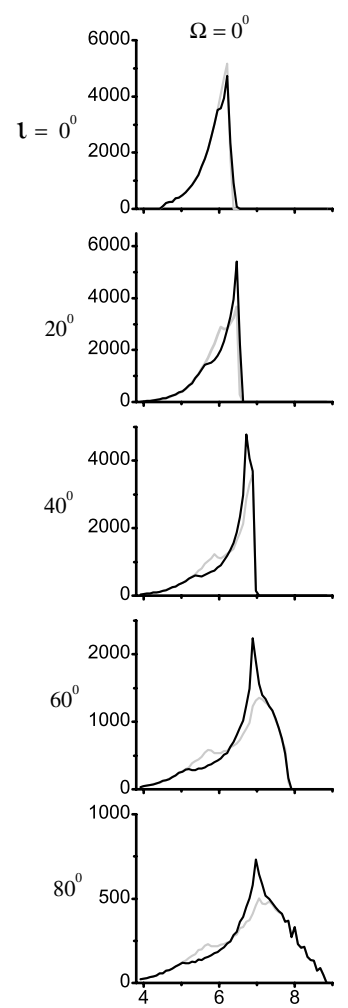
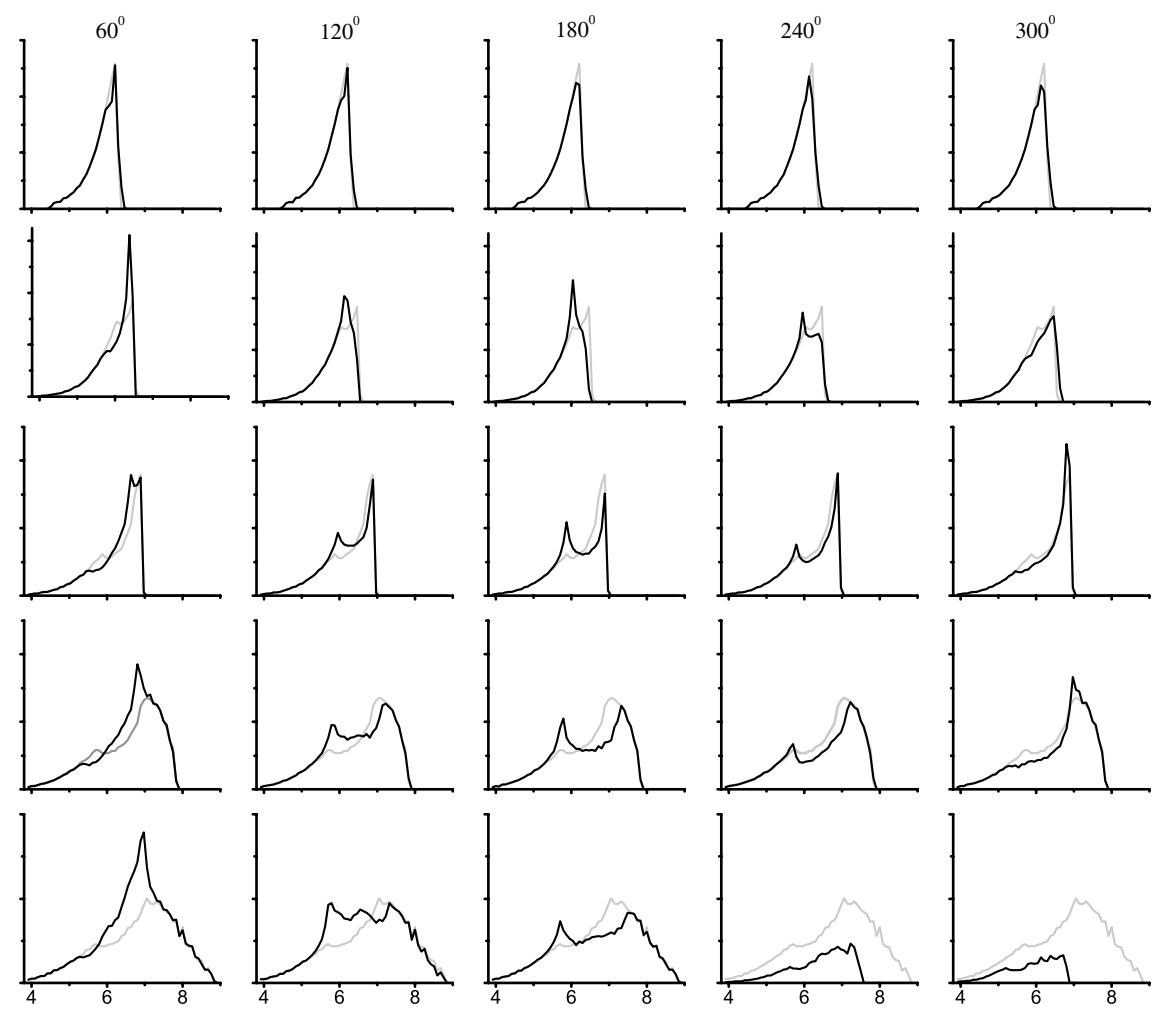

Fig. 3. An array of line profiles of a moderately warped disk $\left(a_{1}=0.1, b=3, R_{\text {out }}=100 M\right)$ together with flat disk line profiles (in light gray) at the same inclination. $X$-axis scale is Energy in keV. $Y$-scale is observed flux in arbitrary units. 

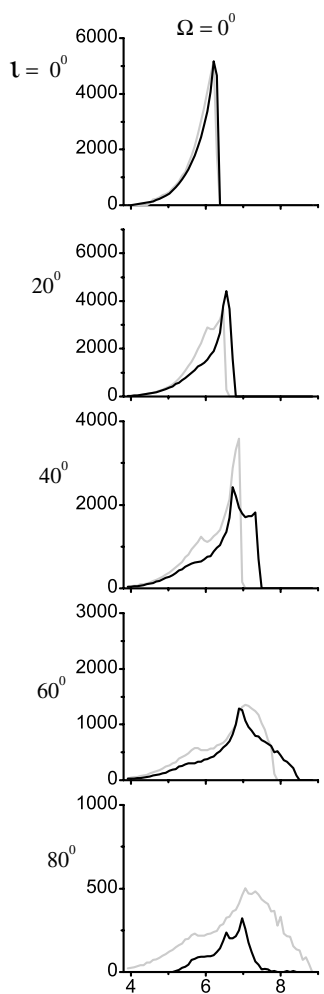
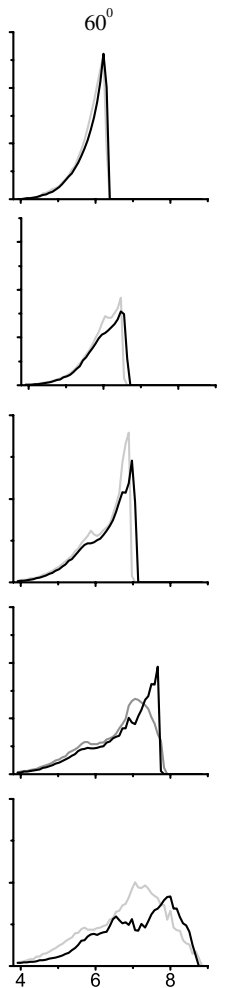
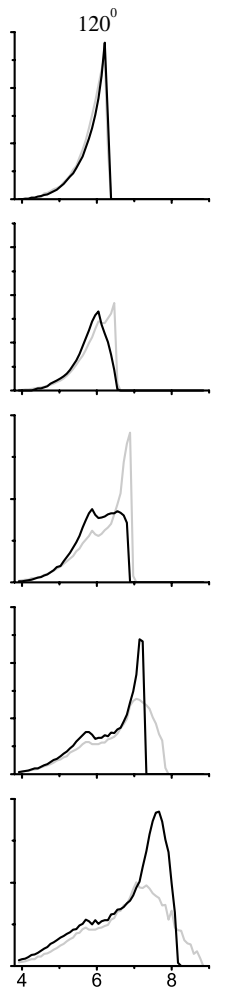
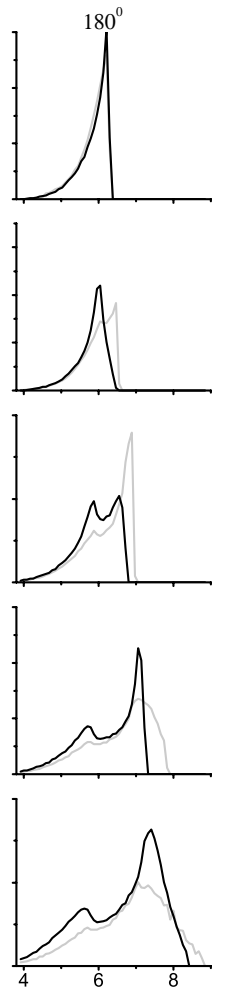
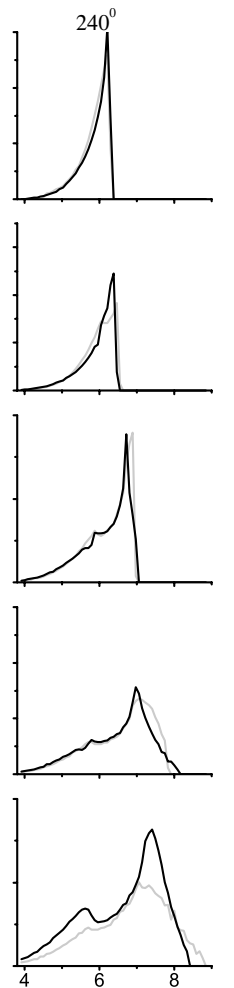
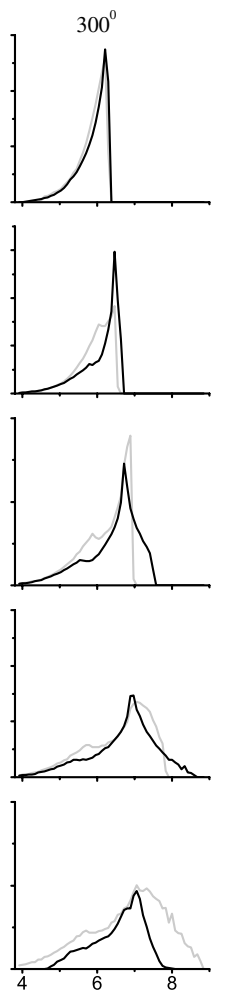

Fig. 4. An array of twisted disk $\left(a_{2}=0.3, R_{\text {out }}=100 M\right)$ line profiles together with flat disk line profiles (in light gray). $X$-axis scale is Energy in keV. $Y$-scale is observed flux in arbitrary units.

profile to the red required a rapidly spinning central black hole (see e.g. Iwasawa et al. 1996; Wilms et al. 2001). However, the emissivity profiles resulting from best fits are not smooth, so that, based on this analysis, we could not strengthen the case for the power emissivity law. In fact, this analysis shows that, with the low signal to noise ratio of the ASCA data, it might be difficult to distinguish between different physical source models.

With the better data coming from XMM and Chandra it becomes important to understand how different emission models leave their mark on the emission line profile. To illuminate this question, we study different theoretical line profiles based on flat and warped disk models with different illumination scenarios. We analyze all these lines as if they belong to a flat disk and try to determine from the success of the fit at what level and how is it possible to determine that a given line does not belong to the flat disk model with a smooth emissivity profile.

The basis of the first example is a flat disk of radius $100 \mathrm{M}$ about a non-rotating black hole, illuminated by two point sources on the axis at $\pm 10 M$ above and below the disk plane. We calculate the appropriate theoretical line profile $F_{0}(E)$ for the inclination $\iota_{0}=30^{\circ}$ and construct noisy lines so that $F(E)=F_{0}(E)+\operatorname{Noise}(\sigma)$, where Noise $(\sigma)$ is computer generated random noise with the same intensity in all energy channels and whose rms fluctuations are denoted by $\sigma$. When solving Eq. (29), we get a solution for $\varepsilon(r)$ depending on the input parameters $\iota_{m}$ and $a_{\text {rot }}$ and also the $\chi^{2}$ of the fit belonging to the difference between the input line profile $F(E)$ and the line profile reconstructed from $\varepsilon(r)$. We select $a_{\text {rot }}=0$ and plot $\chi^{2}$ as a function of $\sigma$ and $\iota_{m}$ in Fig. 5. $\chi^{2}$ is minimal for $\iota_{\min }=30^{\circ}=\iota_{0}$, so one concludes that the inversion procedure finds the correct inclination even if the signal is scrambled by relatively high noise up to about $\sigma>0.06 \times F_{0 \max }$, when the minimum becomes quite wide.

The effect of noise on the line profile, the fit to the line profile and to the calculated emissivity are shown in Fig. 6. The left hand side of the figure shows the original (noise free) profile, the emissivity calculated by solving Eq. (29) for $\iota_{m}=\iota_{\text {min }}$ and the fit to the line calculated from the obtained emissivity (on top of the theoretical line profile). The right hand side of this figure shows the same quantities for the line profile that was contaminated by $3 \%$ noise. It is apparent that the noise free line profile reproduces the emissivity profile of the input model, which in turn reproduces the line profile quite well. The quality of the fit is only mildly dependent on numerical parameters, such as the number and distribution of the radial grid points used in solving Eq. (29). The slight noise noticeable in the emissivity profile comes from numerical noise in producing the line profile.

Note that the emissivity deduced from noisy lines may be quite different from the original noise free emissivity, however, the examples shown seem to indicate that a smooth input emissivity does not generally produce a very jagged output. We understand the former as a consequence of the fact that Eq. (29) does not have a unique solution for $\varepsilon(r)$, since the kernel $K\left(E, R \mid \iota, a_{\text {rot }}\right)$ admits a subspace of solutions of the homogeneous part of Eq. (29). Additional conditions may thus be specified to narrow down the solution space. In our case there is an additional requirement that $\varepsilon(r)>0$ for all $r$. However, as this is a highly nonlinear condition, it is not easy to see 


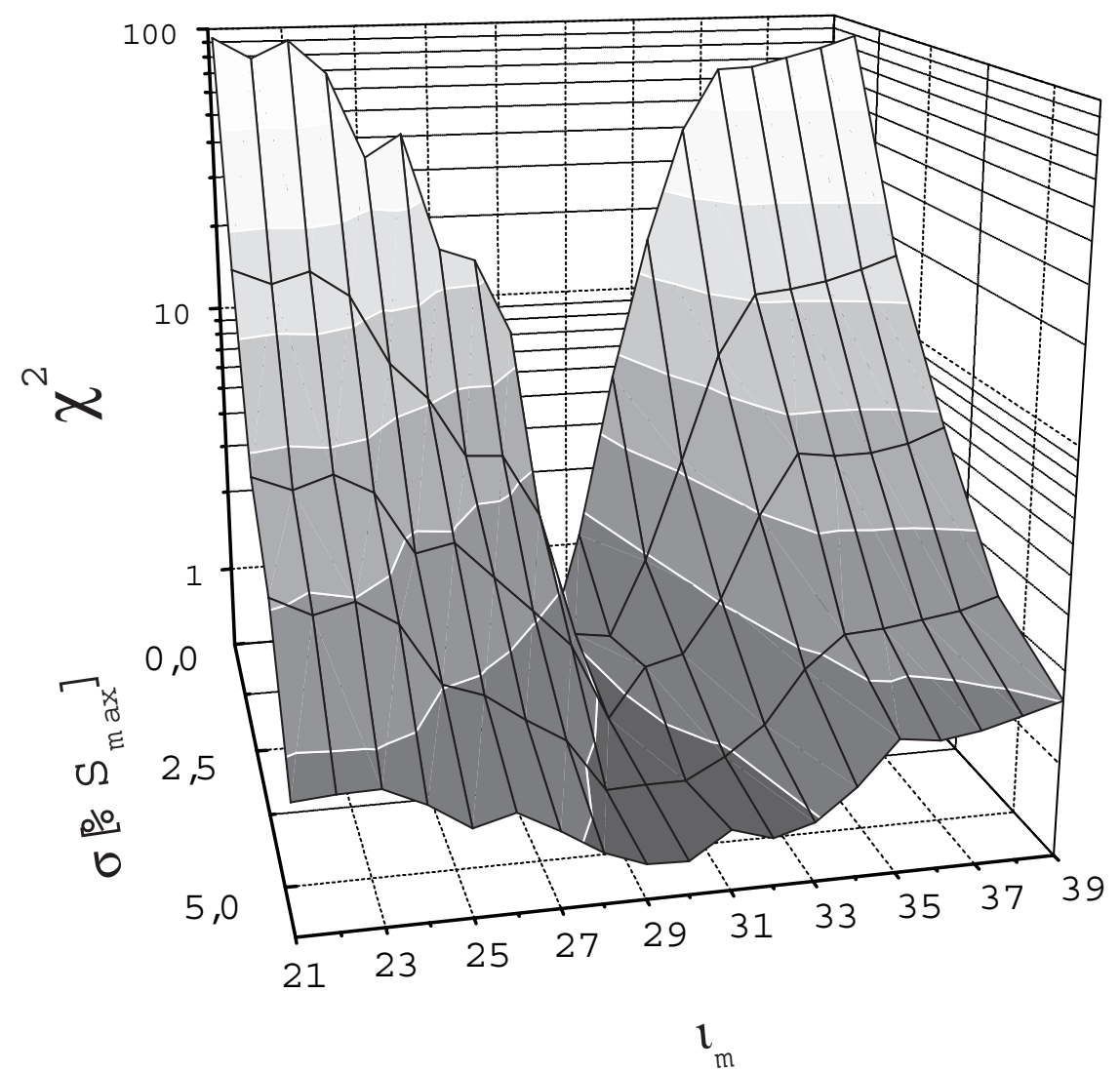

Fig. 5. Fitting the flat disk (at inclination $\iota_{0}=30^{\circ}$ ) noisy line profiles. The $\chi^{2}$ of the fit is shown as function of $\iota_{m}$ and the $\sigma$ of the noise, where $\sigma$ is measured in $\%$ of the maximum signal value. Note that the minimum is always at $30^{\circ}$, but its depth decreases rapidly with $\sigma$.
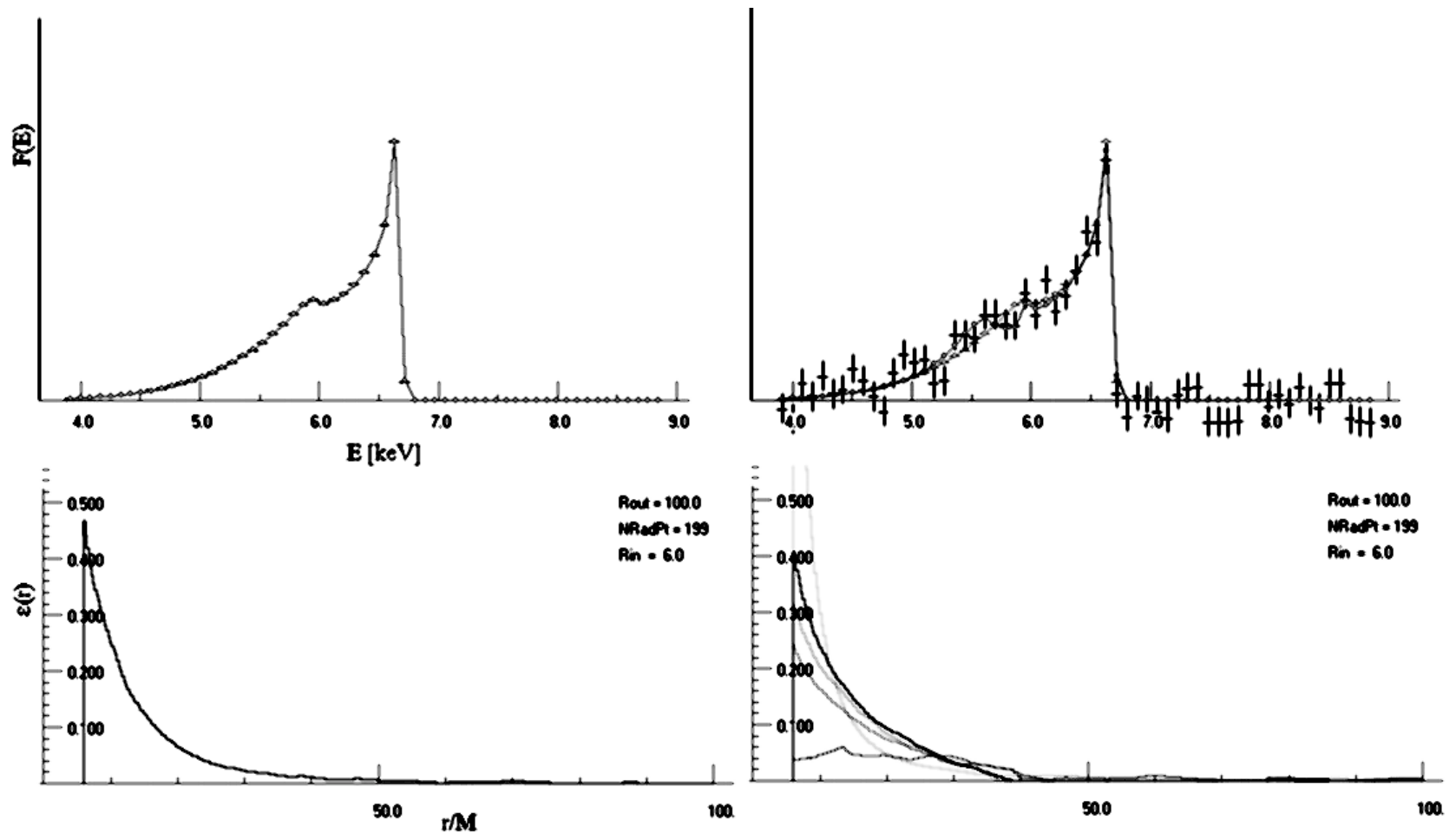

Fig. 6. The line profile and the fit (above) and the calculated emissivity profile (below). Left: theoretical line profile, right: line profile contaminated by $3 \%$ noise. The right top figure shows one random realization of a noisy line; the error bars properly indicate the noise dispersion. The noise free profile is also indicated in light gray. A number of emissivity profiles obtained for different realizations of noise are shown in the right bottom part of the picture, where the heaviest line corresponds to the above line profile. 


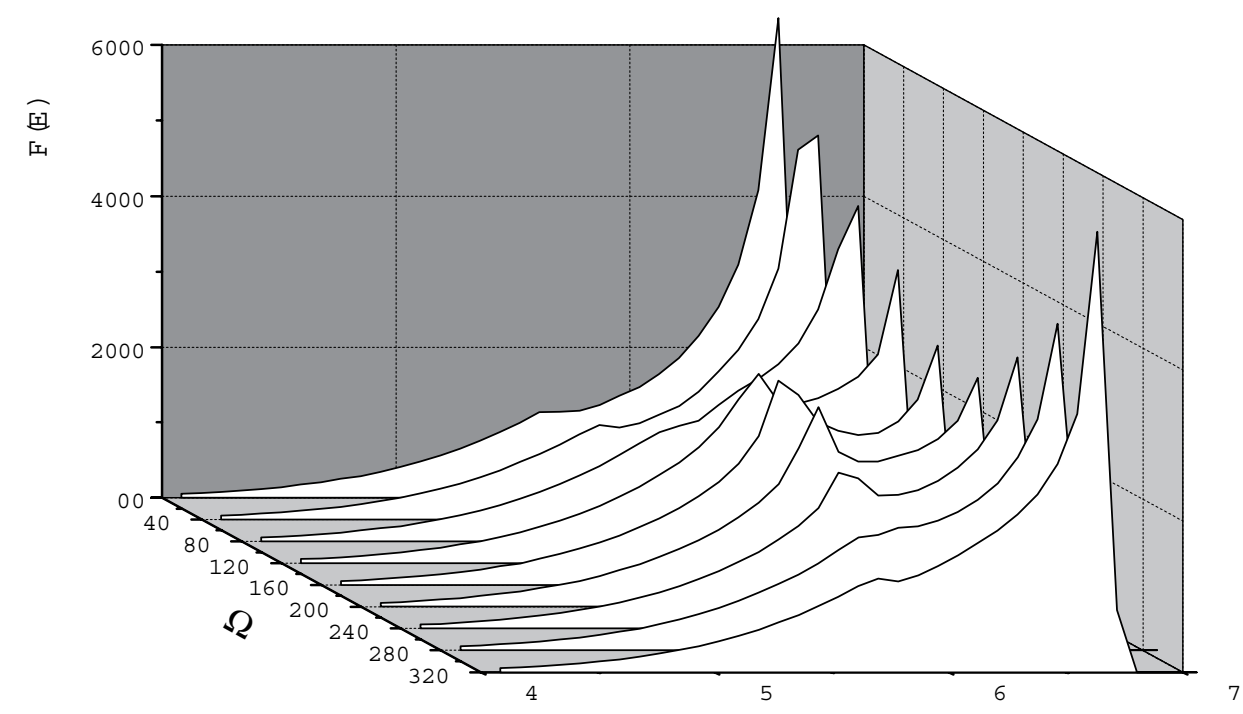

\section{$\mathrm{E} \llbracket \mathrm{keV}]$}

Fig. 7. Line profiles of the $\mathrm{Fe} \mathrm{K}_{\alpha}$ line from a warped disk with $R_{\mathrm{out}}=100 M, a_{\mathrm{rot}}=0, a_{1}=0.06, b=3$ seen at the inclination $\iota=30^{\circ}$ as a function of $\Omega$.

whether it constrains the solution completely. In our variational approach to the solution of Eq. (29) we can apply an additional condition that the length of the curve $\varepsilon(r)$ be minimal. This condition is enforced with a weight parameter $\Lambda$ (see Čadež et al. 2000 for details). Our numerical experience shows that the obtained emissivity profile does not strongly depend on $\Lambda$ as long as it is small (in the appropriate gauge) and the line results in a smooth emissivity profile. However, in general, larger $\Lambda$ produces smoother emissivity profiles at the expense of a worse $\chi^{2}$ of the fit.

The most important lesson of this experiment is learned from the $\chi^{2}$ fits in Fig. 5, which were obtained with $\Lambda=0$. One can see that the width of the $\chi^{2}\left(\iota_{m}\right)$ minimum grows rapidly with increasing noise amplitude $\sigma$. In this numerical experiment $\left(\iota_{0}=30^{\circ}\right)$ we obtain the following relation between the full width of the minimum $\delta \iota$ (measured in degrees) and $\sigma$ (in $\%$ of max value):

$\delta \iota(\sigma) \approx 0.84 \sigma+0.13 \sigma^{2}$.

The parameters of this relationship are, but not in an essential way, dependent on the parameters of the initial theoretical model. This indicates that the subspace of functions that can represent line profiles from flat thin accretion disks has a very small measure, even if all positive solutions of Eq. (29) are allowed. Therefore, this offers a tool to determine if an actual observed line can or can not be considered as belonging to a simple flat disk.

We suggest that a flat disk fit to an observed line with data uncertainty $\sigma$ can be considered as successful only if $\chi^{2} \sim 1$ and also the width of the $\chi^{2}\left(\iota_{m}\right)$ minimum is sufficiently narrow; the relationship (30) gives an approximate measure of this width.

In the next numerical experiment we analyze a series of line profiles belonging to a mildly warped disk with $a_{\text {rot }}=$ $0, a_{1}=0.06, b=3$, illuminated by two point sources at $h= \pm 10 M$. This disk is observed from an inclination $\iota=30^{\circ}$ and the angle $\Omega$ takes on all values. The resulting line profiles as a function of $\Omega$ are shown in Fig. 7, the flat disk model fits in Fig. 8 and the $\chi^{2}$ of the fit to these profiles with the flat disk model are shown in Fig. 9. Here $\chi^{2}$ does not have the usual meaning, as there was no noise intentionally introduced in the data, but it was calculated with the assumption that the mean error of a data point is $10 \%$ of the maximum signal value.

The width of the $\chi^{2}$ minimum is quite narrow, always less than about $2.5^{\circ}$ but its depth depends strongly on the orientation of the warp. It is deepest $\left(\chi^{2} \approx 0.01\right)$ and narrowest at $\Omega=280^{\circ}$, when the warp is seen to bend perpendicular to the line of sight and most shallow $\left(\chi^{2} \approx 0.3\right)$ when the warp is rising toward the observer ${ }^{4}$. Thus, on the basis of Eq. (30) alone it would be possible to accept all line profiles from Fig. 7 as belonging to a flat disk, if data noise were about $2 \%$. However, at this true noise level the actual $\chi^{2}$ would be $(10 / 2)^{2}=25$ times higher, so that at least three out of four line fits would have

${ }^{4}$ This strong $\Omega$ dependence is observed quite generally. On the basis of our numerical experience we propose a simple rule of thumb to account for some distinctions between flat and warped disk line profiles: the warp changes the flat disk line profile by the way in which infinitesimal circular disks, that the disk is made of, are seen. Two components of this aspect are: a) rings are seen at different inclinations due to the way the warp is bent and b) rings are illuminated unevenly due to shading of the light source and due to the varying direction of their normal with respect to the line of sight. Since the most characteristic feature of any ring contribution is the position of the sharp blue edge, the first effect is considerably more important for the success of flat disk fit than the second as long as the blue edge of the ring contribution has not been obliterated by shadowing. Considering a warp that bends perpendicular to the line of sight, we note that all infinitesimal disks have the same inclination with respect to the observer, therefore, their line profiles generally appear most similar to line profiles of flat disks. 


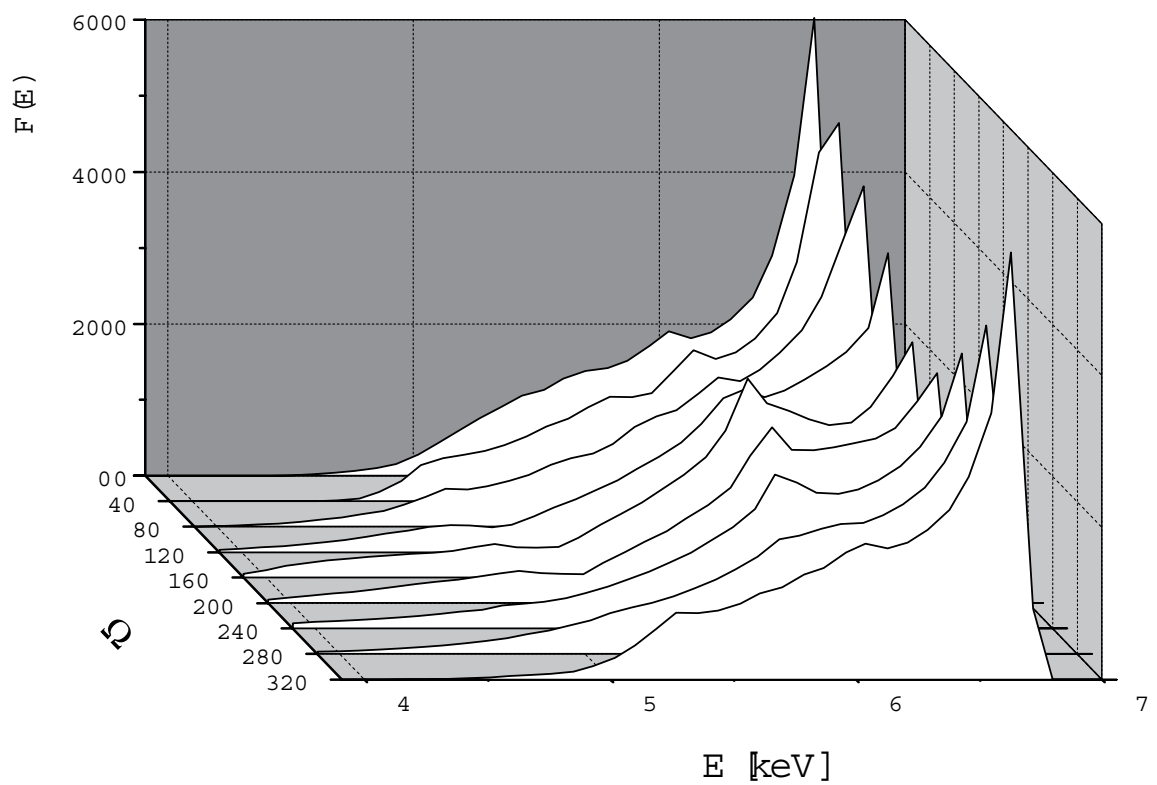

Fig. 8. Line profiles obtained by fitting line profiles shown in Fig. 7 with the flat disk model.

an unacceptable $\chi^{2}$. Repeating the experiment for $a_{\text {rot }}=0$, $a_{1}=0.2, b=3, h= \pm 10 M$, we find that line profiles belonging to $|\Omega|>20^{\circ}$ can not be fitted to a flat disk with acceptable $\chi^{2}$ if the noise level is less than $5 \%$.

The difference between the good and bad fits also appears striking if one observes the emissivity profiles obtained by solving Eq. (29), which are shown in Fig. 10. High $\chi^{2}$ fits generally produce jagged emissivity profiles, while the good ones return the smooth emissivity, which was characteristic of the input model.

On the basis of this experiment we expect that a mildly warped disk with $a_{1}=0.06, b=3, h=10 M$ and $R_{\text {out }}=100 M$ would be accepted as flat only if the warp would happen to be oriented more or less perpendicular to the line of sight, i.e. in a sample of such disks with random orientation of $\Omega$, the warp would avoid detection in only one case out of four, if the noise was less than $2 \%$ of the maximum signal.

The effect of increasing the warp is considered in the last numerical experiment. We again choose a disk with $R_{\text {out }}=$ $100 M$ and $b=3, \iota=30^{\circ}, \Omega=0$ and increase the warp $\left(a_{1}\right)$ in steps of 0.1. The resulting line profiles are shown in Fig. 11, the flat disk model fits in Fig. 12, and the $\chi^{2}$ of the fit (assuming again that $\sigma=10 \%$ ) with respect to $\iota_{m}$ and $a_{1}$ is shown in Fig. 13. It is clear that the depth of the $\chi^{2}$ minimum decreases with increasing warp: for $a_{1}>0.1$ it can be approximated as $\chi_{\text {min }}^{2} \approx 0.8 a_{1}+0.32$. Notice, however, that the low $\iota_{m}$ side of the $\chi^{2}$ surface has "banks" with ridges and valleys reaching as deep as $\chi^{2} \approx 1.2$. Therefore, for warps where the $\chi^{2}\left(\iota_{m}\right)$ minimum is as high as this value, the minimum has no significance and one should conclude that in this case the fit of the line to any flat disc model is unsuccessful. For $\Omega=0$ (which is the aspect of the disk that is most similar to that of a flat disk) this happens at $a_{1}=0.35$.

Figure 14 suggests, as before, that the less successful fits produce jagged emissivity profiles. If found in real data, such jaggedness might be interpreted as non plausible.

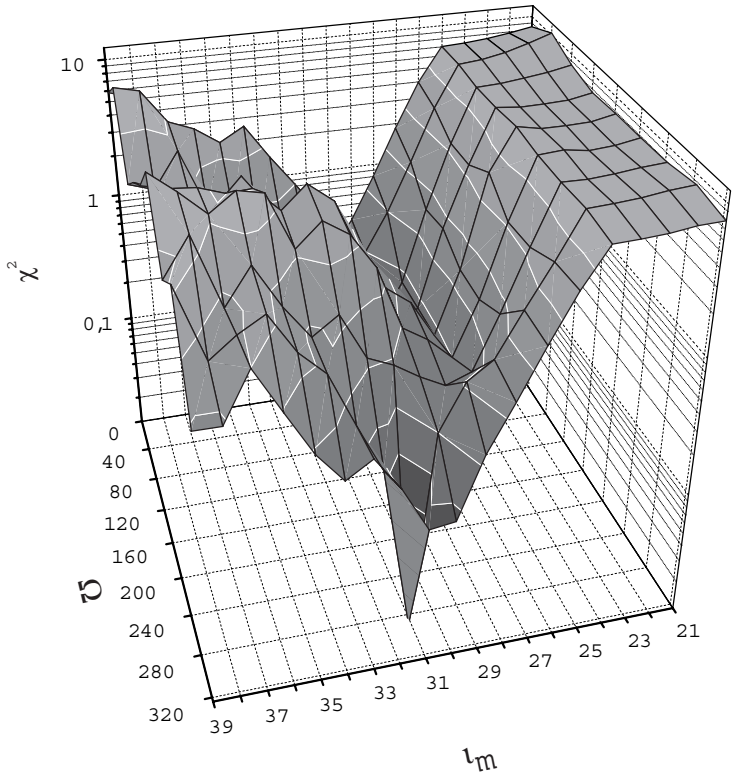

Fig. 9. Flat disk model fits to the line profiles shown in Fig. 7.

The message from the above numerical experiments is very simple: the space of functions belonging to flat disk line profiles is very narrow. This means that in the space of all possible line profiles, the measure of the set of those that belong to the flat disk, irrespective of the radial emissivity, is close to zero. Thus, line profiles produced by warped accretion disks can be distinguished from those of the flat ones if noise in data is sufficiently low.

\section{Rotating black holes: The Kerr metric}

The considerations and results we developed so far are limited to non-rotating black holes. However, at least in interpreting the MCG-6-30-15 X-ray data, a rotating black hole 


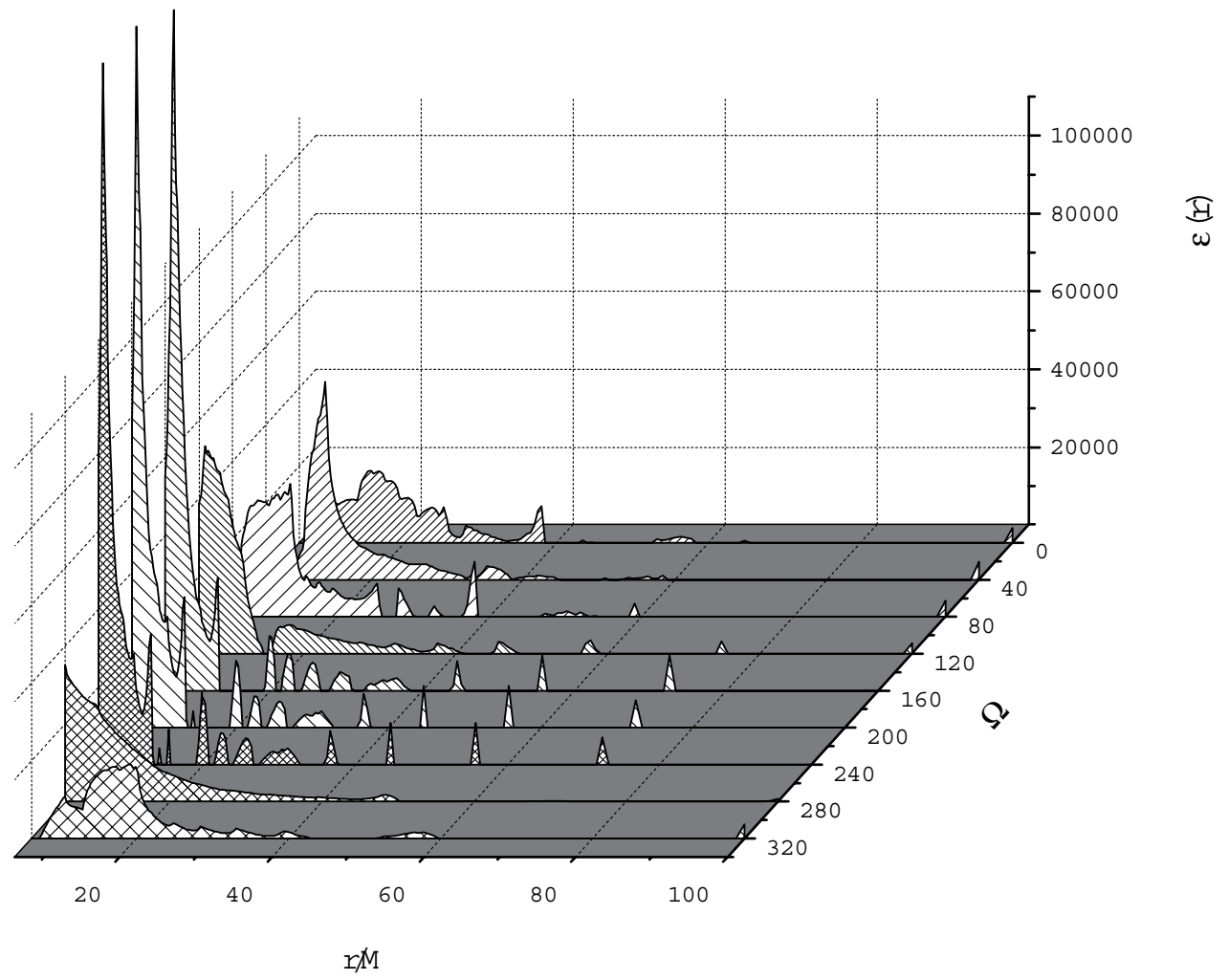

Fig. 10. Emissivity profiles derived for the line profiles shown in Fig. 7.

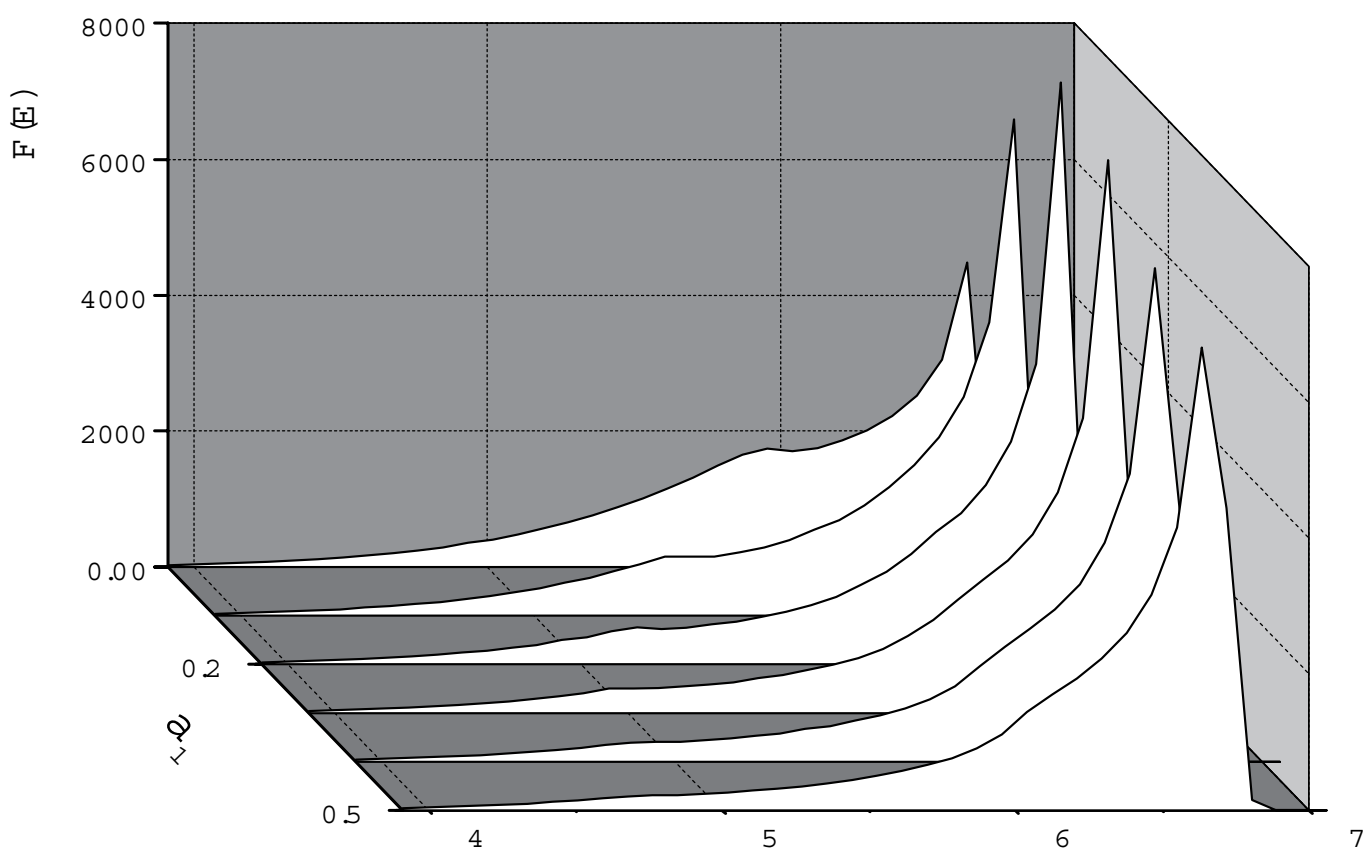

$\mathrm{E}$ [keV]

Fig. 11. The effect of increasing warp on the line profile for a disk with $R_{\text {out }}=100 M, b=3, \iota=30^{\circ}$ and $\Omega=0$. The warp $a_{1}$ is increased in steps of 0.1.

is often invoked (Iwasawa et al. 1996; Sulentic et al. 1998; Lee et al. 2002), while evidence for a warped disk may be Guainazzi et al. 1999; Pariev et al. 2001; Wilms et al. 2001; present in RE J1034+396 (Puchnarewicz \& Soria 2002). 


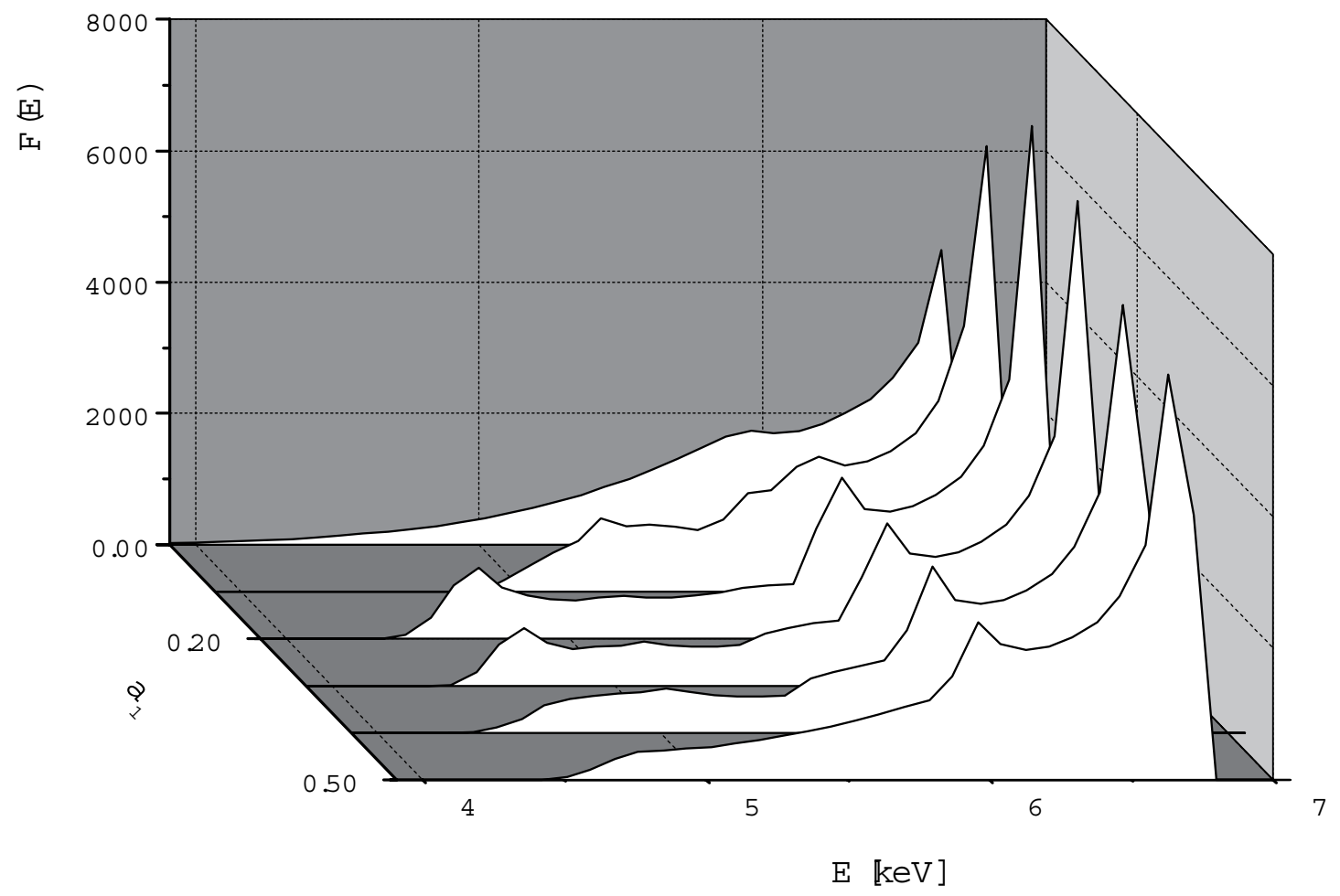

Fig. 12. Line profiles obtained by fitting the profiles shown in Fig. 11 with the flat disk model.

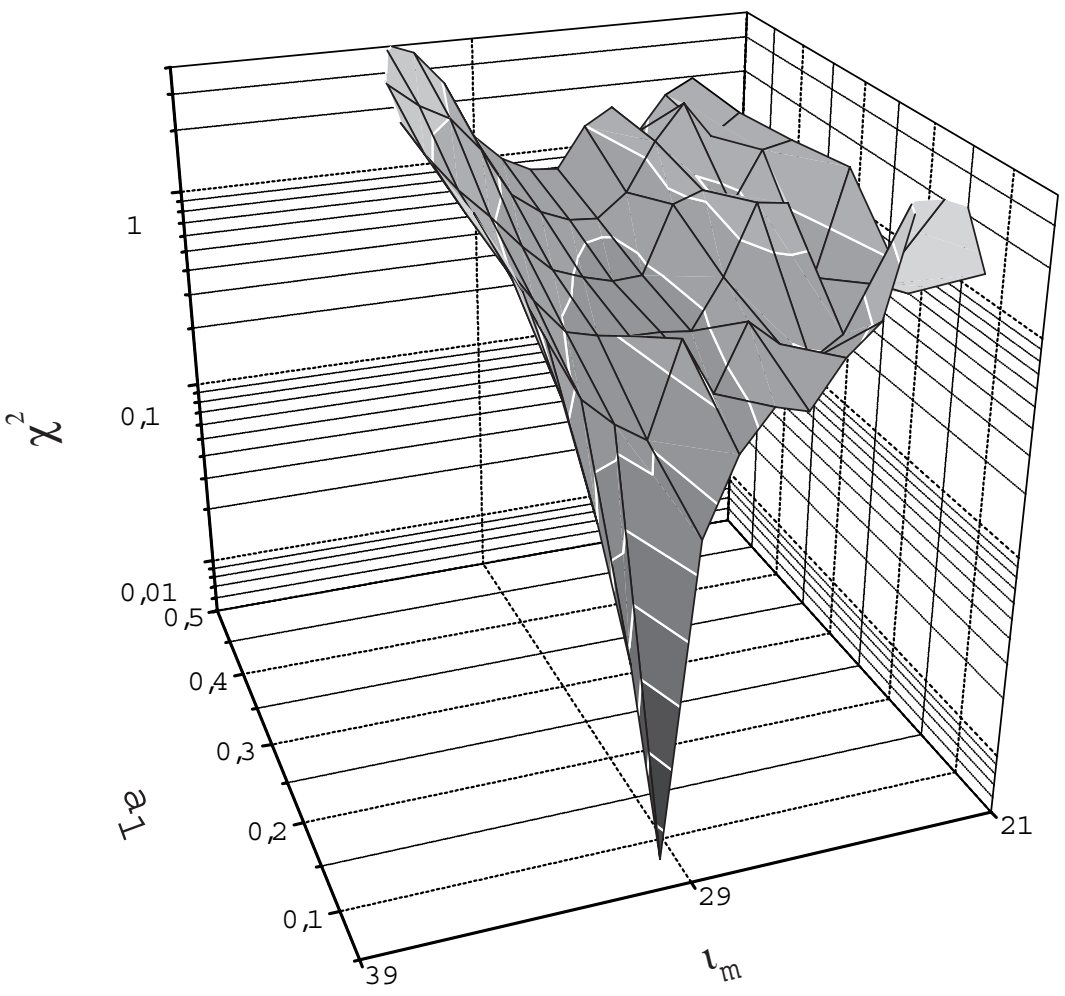

Fig. 13. Flat disk model fits to the line profiles shown in Fig. 11.

The extension of our calculations to warped disks around rotating black holes (Kerr metric) is non trivial for the following reasons: 1) the solutions of geodesic equations cannot (yet?) be written in a form as compact as Eqs. (4) and (12), so that only the map $\mathrm{M}^{-1}$ (and not directly $\mathrm{M}$ ) can be calculated and 2) shearing due to Lense-Thirring precession shapes the geometric form of the inner disk (Bardeen \& Peterson 1975) and determines its rotational law in an essential way. Therefore, a meaningful disk model would have to be derived from a consistent integration of the accretion flow dynamic equations. 


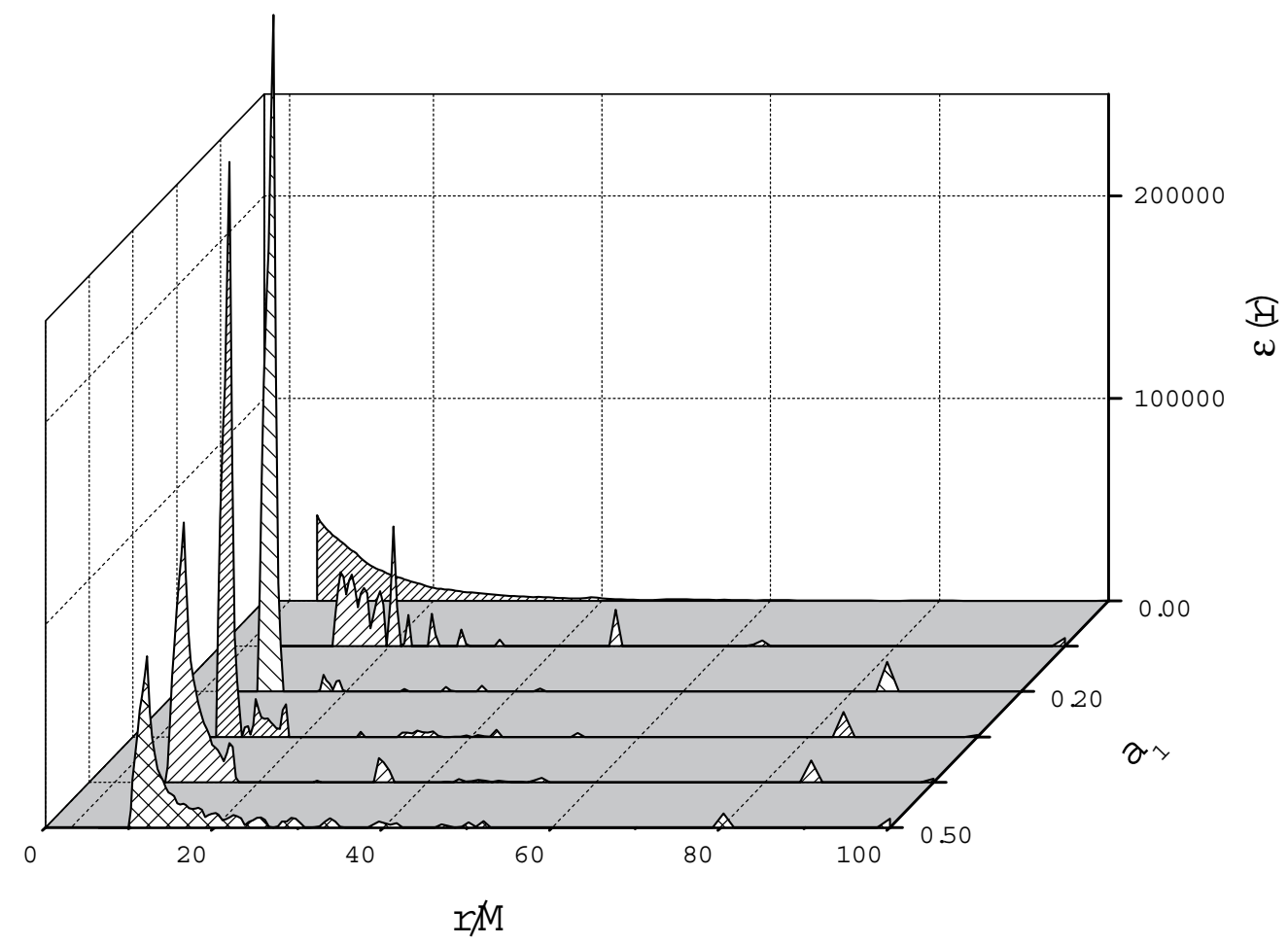

Fig. 14. Emissivity profiles derived for the line profiles shown in Fig. 11.

Using some simplifying assumptions about the shape and the angular velocity profile of the warped disk around a Kerr black hole, we are able to compute the line profiles, some examples of which are shown in Fig. 15 (Čadež et al. 2002). The program solves the equations of motion analytically: we decompose the $r, \theta, \phi$ components of these equations into a combination of elliptic and elementary integrals. The program uses a ray-tracing technique: starting from any point on the photographic plate (we consider the observer placed above the equatorial plane) it calculates the constants of motion of the photon trajectory and then it finds the intersection of the trajectory with the equatorial plane. Starting from this point, the program looks for the intersection of the trajectory with the warped disc; the photon trajectory is then evaluated upward and downward. If there is an intersection above the equatorial plane, the program stops the integration: in fact any other intersection below the equatorial plane corresponds to a shadowed region of the disc. In general, if there are multiple intersections between the trajectory and the warped disc, the program considers the one at highest $z$-coordinate: the other ones are associated to points that are not seen from the distant observer. This procedure is repeated for each trajectory computed by the program.

As expected, the main difference from the Schwarzschild case is the larger extent of the red wing of the line. However the conclusion that the space of functions belonging to flat disk line profiles is very narrow, will still hold.

\section{Conclusions}

The original close form analytic solutions presented in Sect. 2 are very efficient as a tool in applying ray-tracing techniques in the curved space-time of non-rotating black holes. Using this tool we were able to calculate a large number of images and line profiles belonging to theoretical warped accretion disks around non-rotating black holes. The same tool is also found very useful for calculating the kernel of the integral equation which connects the disk emissivity to the line profile, in the framework of the flat accretion disk model, as discussed in Čadež et al. (2000). Using these tools we performed a deep investigation of line profiles from warped disks around non-rotating black holes and compared them to those belonging to flat disks. As a result, we find that: 1) the space of functions belonging to flat disk line profiles is very narrow. This means that in the space of all possible line profiles, the measure of the set of those belonging to the flat disk, irrespective of the radial emissivity, is close to zero. 2) On this basis, we propose a method to distinguish between line profiles that do belong to thin flat disks and those that do not. We expect that this may become a useful tool as soon as better, higher $S / N \mathrm{X}$-ray data will be available.

We also presented some results for warped disks around rotating black holes, and they point to the same general conclusion that lines belonging to flat disks form a very narrow subspace in the space of functions that describe all possible accretion disks.

We point out that a different geometry (such as multiple disk flares, or a disk covering or a patchy corona) could complicate the issue, but we are confident that the above results will still hold.

Acknowledgements. The work of AČ and AG was supported by the research grant of the Ministry of Science, Education and Sport of the RS. MC acknowledges the financial support of the Italian MIUR through grant Cofin 00-02-004. 

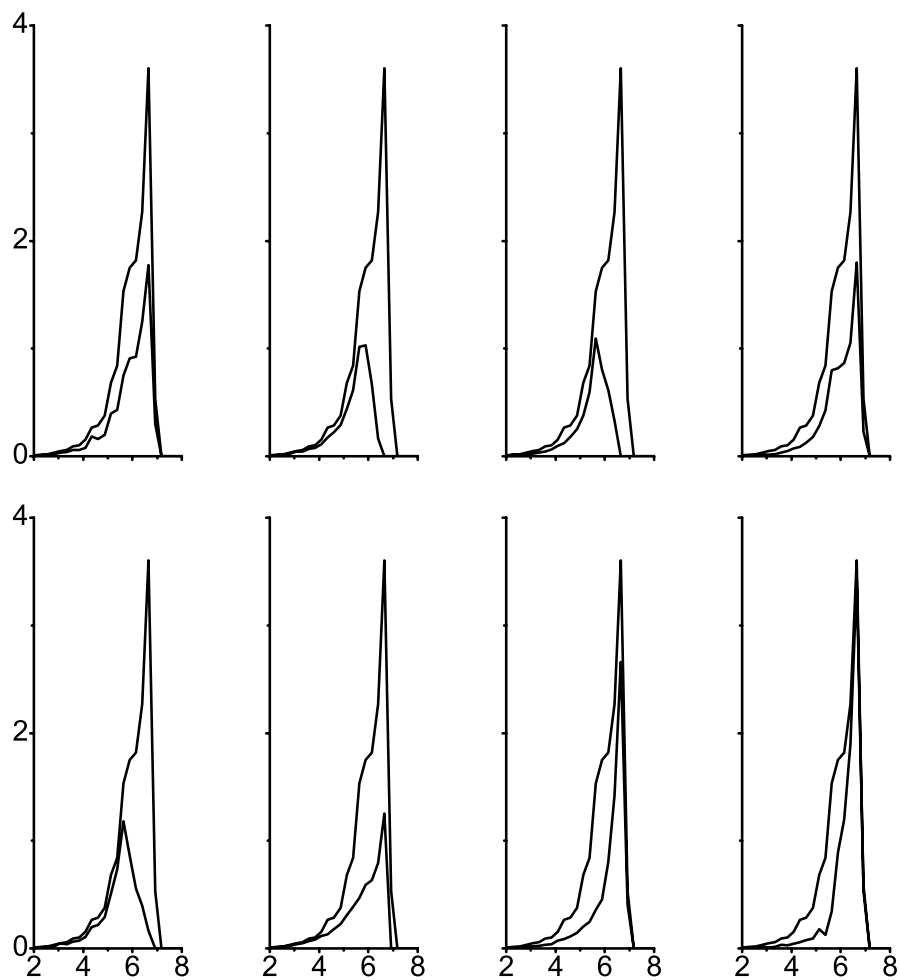
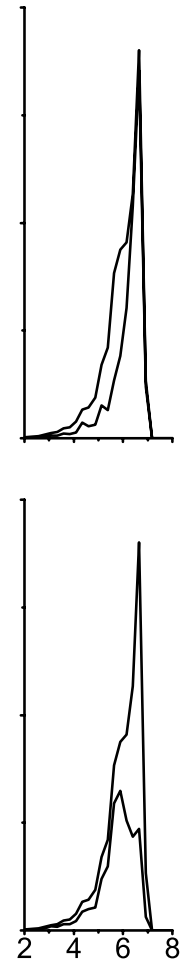

Fig. 15. Some examples of line profiles for warped disks around a rotating (Kerr) black hole with $a / M=0.009981$. All profiles (thick lines) are computed for $\iota=40^{\circ}, R_{\text {out }}=100 \mathrm{M}$. Top row: twist free disk; bottom row: twisted disk. In each row, profiles are arranged in order of increasing $\Omega$, from $\Omega=0^{\circ}$ to $\Omega=300^{\circ}$ in steps of $60^{\circ}$. The emissivity law is $\epsilon(r) \propto r^{-2}$. The thin line represents, for comparison, the line profile for an equatorial (flat) disk for the same inclination and outer radius. $X$-axis scale is Energy in keV. $Y$-scale is observed flux in arbitrary units.

\section{References}

Bachev, R. 1999, A\&A, 348, 71

Bardeen, J. M., \& Peterson, J. A. 1975, ApJ, 195, L65

Blackman, E. G. 1999, MNRAS, 306, L25

Brajnik, M. 1999, BS Thesis, Univ. Ljubljana

Čadež, A., Fanton, C., \& Calvani, M. 1998, New Astron., 3, 647

Čadež, A., Calvani, M., Di Giacomo, C., \& Marziani, P. 2000, New Astron., 5, 69

Čadež, A., Calvani, M., \& Fanton, C. 2001, in Proc. of XLVI Meet. of Italian Astronomical Society SaIt

Calvani, M., Marziani, P., \& Sulentic, J. 1997, Mem. della Soc. Astron. It., 68, 93

Dabrowski, Y., Fabian, A. C., Iwasawa, K., Lasenby, A. N., \& Reynolds, C. S. 1997, MNRAS, 288, L11

Demianski, M., \& Ivanov, P. B. 1997, A\&A, 324, 829

Fabian, C. A., Nandra, K., Reynolds, C. S., et al. 1995, MNRAS, 277, L11

Fabian, A. C., Iwasawa, K., Reynolds, C. S., \& Young, A. J. 2000, PASP, 112,1145

Fabian, A. C. 2001, in X-ray and accretion disks as probes of the strong gravity of black holes, ed. C. Wheeler, \& H. Martel, AIP Conf. Proc., 586

Fanton, C., Calvani, M., de Felice, F., \& Čadež, A. 1997, PASJ, 49, 159

Gomboc, A. 2001, Ph.D. Thesis, Univ. Ljubljana

Guainazzi, M., Matt, G., Molendi, S., et al. 1999, A\&A, 341, L27

Hartnoll, S. A., \& Blackman, E. G. 2000, MNRAS, 317, 880

Hartnoll, S. A., \& Blackman, E. G. 2001, MNRAS, 324, 257

Iwasawa, K., Fabian, A. C., Reynolds, C. S., et al. 1996, MNRAS, 282,1038
Karas, V., Martocchia, A., \& Subr, L. 2001, PASJ, 53, 189

Kojima, Y., \& Fukue, J. 1992, MNRAS, 256, 679

Lee, J. C., Iwasawa, K., Houck, J. C., et al. 2002, ApJ, 570, L47

Lucy, L. B. 1974, AJ, 79, 745

Lubow, S. H., Ogilvie, G. I., \& Pringle, J. E. 2002 [astro-ph/0208206]

Mannucci, F., Salvati, M., \& Stanga, R. M. 1992, ApJ, 394, 98

Martocchia, A., Karas, V., \& Matt, G. 2000, MNRAS, 312, 817

Martocchia, A., Matt, G., \& Karas, V. 2002, A\&A, 383, L23

Nandra, K., George, I. M., Mushotzki, R. F., Turner, T. J., \& Yaqoob, T. 1997, ApJ, 477, 602

Pariev, V. I., Bromley, B. C., \& Miller, W. A. 2001, ApJ, 547, 649

Pringle, J. E. 1996, MNRAS, 281, 357

Pringle, J. E. 1997, MNRAS, 292, 136

Puchnarewicz, E. M., \& Soria, R. 2002 [astro-ph/0202030]

Quillen, A. C. 2001, ApJ, 563, 313

Reeves, J. N., Pounds, K. A., O’Brien, P. T., \& Turner, M. J. 2001, in X-ray emission from accretion onto black holes, JHU/LHEA Workshop

Reynolds, C. S., \& Nowak, M. A. 2002 [astro-ph/0212065]

Sanbuichi, K., Fukue, J., \& Kojima, Y. 1994, PASJ, 46, 605

Sulentic, J. W., Marziani, P., \& Calvani, M. 1998, ApJ, 497, L65

Sulentic, J. W., Marziani, P., \& Dultzin-Hacyan, D. 2000, ARA\&A, 38,521

Usui, F., Nishida, S., \& Eriguchi, Y 1998, MNRAS, 301, 721

Yu, Q., \& Lu, Y. 2000, MNRAS, 311, 161

Wilms, J., Reynolds, C. S., Begelman, M. C., et al. 2001, MNRAS, 328, L27

Wolfram, S. 1996, The Mathematica book, 3rd ed. (Wolfram media, Cambridge University Press) 